Supporting Information for

\title{
The Effects of Glycine on the Local Conformation and Internal Backbone Dynamics of Polypeptides
}

\author{
Remi Casier and Jean Duhamel*
}

Institute for Polymer Research, Waterloo Institute for Nanotechnology, Department of Chemistry, University of Waterloo, Waterloo, ON, N2L3G1, Canada

*To whom correspondence should be addressed: jduhamel@uwaterloo.ca 


\section{Table of Contents}

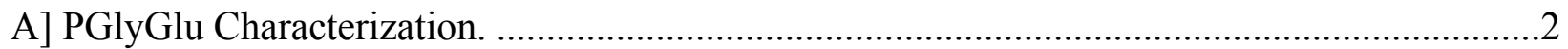

B] Py-PGlyGlu preparation and pyrene content characterization. .............................................

C] Equations used to globally analyze the fluorescence decays of the Py-PGlyGlu samples........4

D] Example global FBM analysis of the monomer and excimer fluorescence decays of

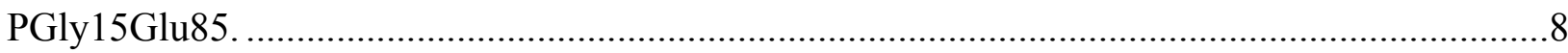

E] Parameters retrieved from the FBM analysis of the pyrene-labeled polypeptides. ..................9

F] Circular Dichroism of the pyrene-labeled polypeptides. ..................................................15

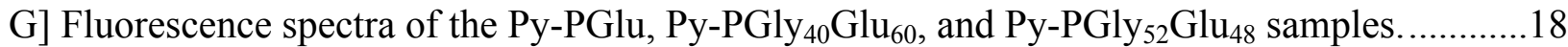

$\mathrm{H}] k_{\text {blob }}$ values of the pyrene-labeled polypeptides......................................................... 19

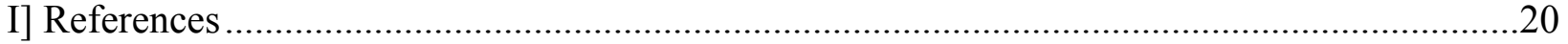


A] PGlyGlu Characterization. PGly ${ }_{40} \mathrm{Glu}_{60}$ and $\mathrm{PGly}_{15} \mathrm{Glu}_{85}$ were synthesized according to a previously reported procedure with only a slight modification in monomer content. ${ }^{1}$ The Gly content $\left(f_{\mathrm{Gly}}\right)$ of $\mathrm{PGly}_{40} \mathrm{Glu}_{60}$ and $\mathrm{PGly}_{15} \mathrm{Glu}_{85}$ were determined from the ${ }^{1} \mathrm{H}$ NMR spectra in Figures $\mathrm{S} 1$ and $\mathrm{S} 2$, respectively, using the relative areas $A$ of the Gly and Glu $\alpha$ protons $\left(f_{\mathrm{Gly}}=\right.$ $\left.A_{\mathrm{Gly}} /\left(A_{\mathrm{Gly}}+2 A_{\mathrm{Glu}}\right)\right)$. The degree of polymerization $(D P)$ was calculated as $D P=3 \times A_{\mathrm{NH}} / A_{\mathrm{I}}$, where $A_{\mathrm{NH}}$ and $A_{\mathrm{I}}$ are the relative areas of the amide and initiator peak, respectively. The number-average molecular weights $\left(M_{\mathrm{n}}\right)$ in the main text were calculated using Equation S1, where $M_{\mathrm{Gly}}(=57.05$ $\mathrm{g} / \mathrm{mol})$ and $M_{\mathrm{Glu}}(=129.12 \mathrm{~g} / \mathrm{mol})$ are the molecular weights of Gly and Glu structural units, respectively.

$$
M_{n}=D P \times\left[f_{G l y} M_{G l y}+\left(1-f_{G l y}\right) M_{G l u}\right]
$$

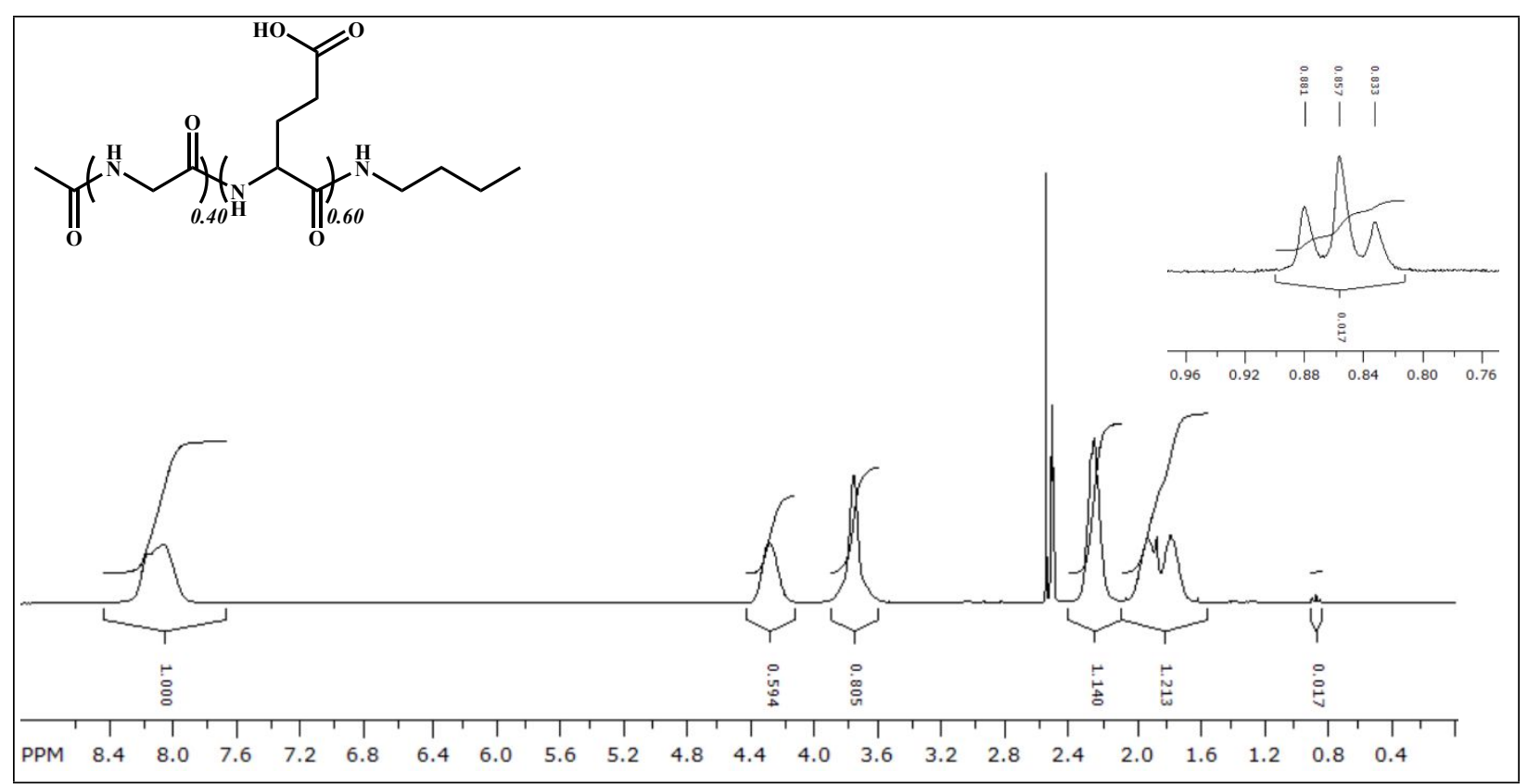

Figure S1. ${ }^{1} \mathrm{H}$ NMR of PGly40Glu60. $300 \mathrm{MHz}, \mathrm{d}_{6}$-DMSO: $\delta=8.09$ (1H, amide), $4.25(2 \mathrm{H}$, $\alpha$-Gly), 3.72 (1H, $\alpha$-Glu), 2.23 (2H, $\gamma$-Glu), $1.88-1.73$ (2H, $\beta$-Glu), $1.25-1.21$ (m, 4H), 0.86 (t, 
$3 \mathrm{H}$, initiator peak), and $1.36(9 \mathrm{H}, \mathrm{tBu}) \mathrm{ppm}$. Residual water $(3.31 \mathrm{ppm})$ and solvent $(2.47 \mathrm{ppm})$ peaks are also present. The methyl peak of the $n$-butylamine initiator is enlarged as an inlay.

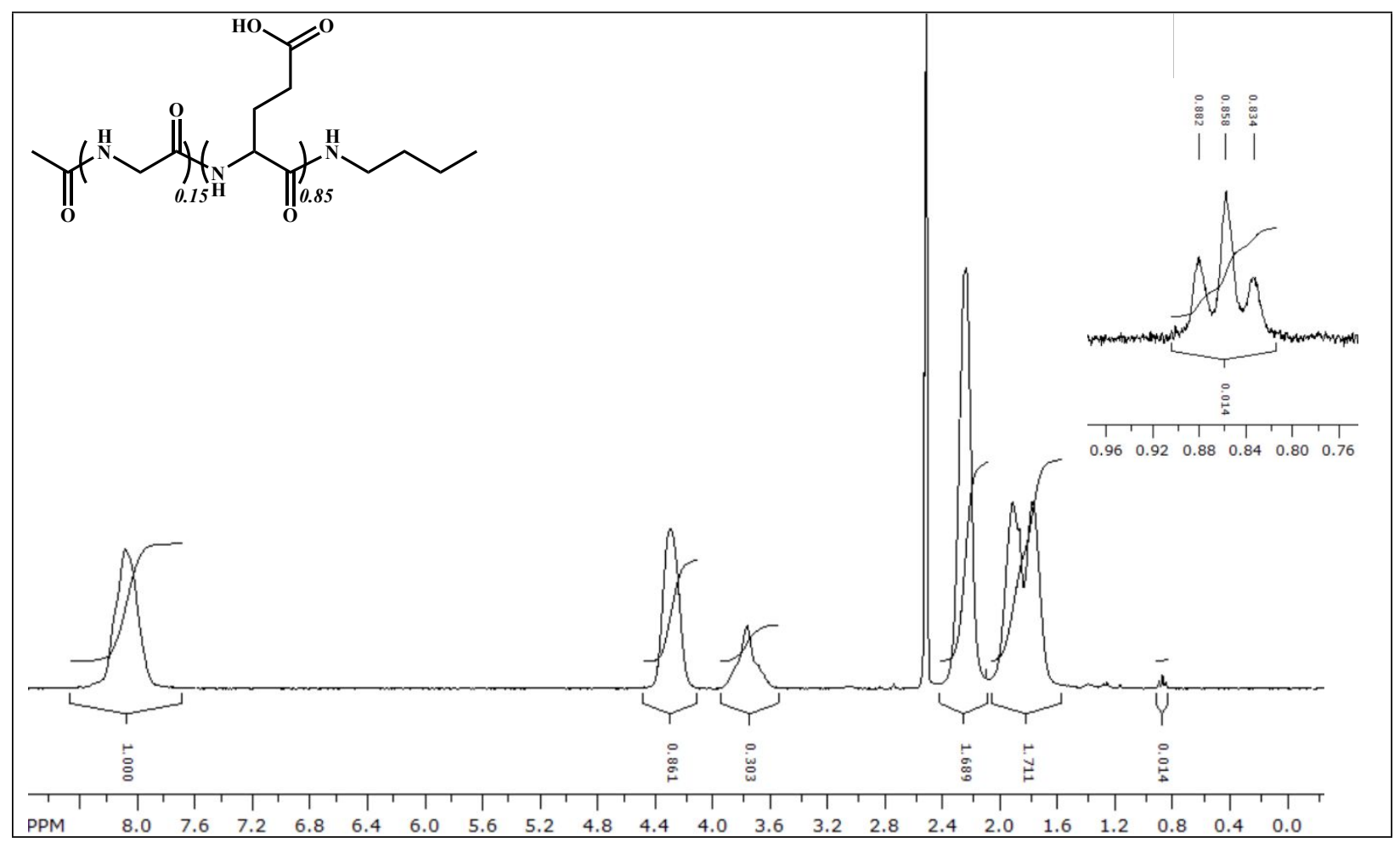

Figure S2. ${ }^{1} \mathrm{H}$ NMR of PGly15Glu85. $300 \mathrm{MHz}, \mathrm{d}_{6}$-DMSO: $\delta=8.09(1 \mathrm{H}$, amide), $4.25(2 \mathrm{H}$,

$\alpha$-Gly), 3.72 (1H, $\alpha$-Glu), 2.23 (2H, $\gamma$-Glu), $1.88-1.73$ (2H, $\beta$-Glu), $1.25-1.21$ (m, 4H), $0.86(\mathrm{t}$, $3 \mathrm{H})$, and $1.36(9 \mathrm{H}, \mathrm{tBu}) \mathrm{ppm}$. Residual water $(3.31 \mathrm{ppm})$ and solvent $(2.47 \mathrm{ppm})$ peaks are also present. The methyl peak of the $n$-butylamine initiator is enlarged as an inlay.

B] Py-PGlyGlu preparation and pyrene content characterization. The Py-PGlyGlu samples were labeled with 1-pyrenemthylamine according to a previously described procedure. ${ }^{1}$ The pyrene-labeled polypeptides were then purified by dialysis. The samples were dialyzed twice for at least 4 hours against $400 \mathrm{~mL}$ of DMF containing ca. $1 \mathrm{~mL}$ of $1 \mathrm{M} \mathrm{HCl}$ to remove any unbound pyrene. The solvent was then gradually changed to water by dialyzing against $400 \mathrm{~mL}$ solutions of 1:3, 1:1, 3:1 water:DMF mixtures. Lastly, the remaining DMF was removed by dialyzing three 
times against $400 \mathrm{~mL}$ water with $\mathrm{HCl}$ ( $\mathrm{pH} \sim 2$, Hydrion Insta-Check $\mathrm{pH}$ test paper). Py-PGlyGlu with its protonated glutamic acid side chains was then recovered by freeze-drying the aqueous solution (ca.10 mL). To ensure that the dialyzed solution contained a negligible amount of salt, a $20 \mathrm{~mL}$ aliquot of the equilibrated dialysate was also freeze-dried, which left no remnants.

The pyrene content of the salt-free Py-PGlyGlu samples were then determined as follows. A DMSO solution containing a known mass concentration $\left(m_{\text {Poly }}\right)$ of Py-PGlyGlu was prepared. The molar concentration $\left(C_{\mathrm{Py}}\right)$ of pyrene in the solution was determined by applying the BeerLambert law to the background-corrected UV-Vis absorbance, determined with a Varian Cary 100 Bio spectrophotometer, of the Py-PGlyGlu solution at $346.5 \mathrm{~nm}$ using the molar absorptivity coefficient $(\varepsilon)$ of the model compound $N$-(1-pyrenylmethyl)acetamide (PyMAAc) in DMSO $\left(\varepsilon(346.5 \mathrm{~nm})=39,300 \mathrm{M}^{-1} \cdot \mathrm{cm}^{-1}\right) .^{2}$ The moles of pyrene per gram of polypeptide $\left(\lambda_{\mathrm{Py}}=C_{\mathrm{Py}} / m_{\text {Poly }}\right)$ was then substituted into Equation S2 to determine the molar fraction $(x)$ of structural units labeled with pyrene. In Equation $\mathrm{S} 2, f_{\mathrm{Gly}}$ is the Gly content determined by ${ }^{1} \mathrm{H}$ NMR and $M_{\mathrm{Gly}}(=57.05$ $\mathrm{g} / \mathrm{mol}), M_{\mathrm{Glu}}(=129.12 \mathrm{~g} / \mathrm{mol})$, and $M_{\mathrm{Py}}(=342.4 \mathrm{~g} / \mathrm{mol})$ are the molar masses of the Gly, Glu, and pyrene-labeled Glu structural units, respectively.

$$
x=\frac{f_{G l y} M_{G l y}+\left(1-f_{G l y}\right) M_{G l u}}{\lambda_{P y}{ }^{-1}+M_{G l u}-M_{P y}}
$$

\section{C] Equations used to globally analyze the fluorescence decays of the Py-PGlyGlu samples.}

The monomer and excimer fluorescence decays were globally analyzed using Equations S3 and $\mathrm{S} 4$, respectively. Briefly, the overall monomer emission combines the fluorescence of the pyrene species $P y_{\mathrm{k} 2}{ }^{*}$, which undergo a rapid rearrangement with a nearby ground-state pyrene with a rate constant $k_{2}$ to form an excimer, $P y_{\text {diff }}{ }^{*}$, that must first diffuse inside a $b l o b$ with a rate constant $k_{\text {blob }}$ 
before arriving in close contact with a ground-state pyrene and transform into $P y_{\mathrm{k} 2}{ }^{*}$, and $P y_{\mathrm{free}}{ }^{*}$, which do not form excimer and emit with their natural lifetime $\tau_{\mathrm{M}}$. The excimer decay contains the emission of the pyrene species, which form the excimers $E 0^{*}$ and $D^{*}$, which emit with their lifetimes $\tau_{\mathrm{E} 0}$ and $\tau_{\mathrm{D}}$, respectively. The excimer species are formed through the sequential process beginning with $P y_{\text {diff }}{ }^{*}$ transforming into $P y_{\mathrm{k} 2}{ }^{*}$, and turning into an excimer and through direct excitation of ground-state pyrene aggregates $\left([E 0]_{0}\right.$ and $\left.[D]_{0}\right)$. Ground-state pyrenes can also exchange between the blobs with the rate $k_{\mathrm{e}}[b l o b]$, where $k_{\mathrm{e}}$ is the rate constant for exchange between blobs and $[b l o b]$ is the local concentration of blobs in the polymer coil. The average number of pyrenes per $b l o b$ is equal to $<n>$.

$$
\begin{aligned}
& {\left[P y^{*}\right]_{(t)}=\left[P y_{\text {diff }}^{*}\right]_{(t)}+\left[P y_{k 2}^{*}\right]_{(t)}+\left[P y_{\text {free }}^{*}\right]_{(t)}=\left[P y_{\text {diff }}^{*}\right]_{o} \exp \left(-\left(A_{2}+\frac{1}{\tau_{M}}\right) t-A_{3}\left(1-\exp \left(-A_{4} t\right)\right)\right) }+\left(\left[P y_{k 2}^{*}\right]_{o}+\left[P y_{\text {diff }}^{*}\right]_{o} e^{-A_{3}} \sum_{i=0}^{\infty} \frac{A_{3}^{i}}{i !} \frac{A_{2}+i A_{4}}{A_{2}+i A_{4}-k_{2}}\right) \exp \left(-\left(k_{2}+\frac{1}{\tau_{M}}\right) t\right) \\
&-\left[P y_{\text {diff }}^{*}\right]_{o} e^{-A_{3}} \sum_{i=0}^{\infty} \frac{A_{3}^{i}}{i !} \frac{A_{2}+i A_{4}}{A_{2}+i A_{4}-k_{2}} \exp \left(-\left(A_{2}+i A_{4}+\frac{1}{\tau_{M}}\right) t\right) \\
& {\left[E^{*}\right]_{(t)}=\left[E 0^{*}\right]_{(t)}+\left[D^{*}\right]_{(t)}=k_{2}\left(\left[P y_{k 2}(E 0)\right]_{o}+\left[P y_{\text {diff }}(E 0)\right]_{o} e^{-A_{3}} \sum_{i=0}^{\infty} \frac{A_{3}^{i}}{i !} \frac{A_{2}+i A_{4}}{A_{2}+i A_{4}-k_{2}}\right) } \\
&+\left[P y_{\text {free }}^{*}\right]_{o} \exp \left(-\frac{t}{\tau_{M}}\right) \\
& \quad \frac{\exp \left(-\frac{t}{\tau_{E 0}}\right)-\exp \left(-\left(k_{2}+\frac{1}{\tau_{M}}\right) t\right)}{k_{2}+\frac{1}{\tau_{M}}-\frac{1}{\tau_{E 0}}}
\end{aligned}
$$




$$
\begin{aligned}
& \left.+\left[P y_{\text {diff }}(E 0)\right]_{o} e^{-A_{3}} \sum_{i=0}^{\infty} \frac{A_{3}^{i}}{i !} \frac{A_{2}+i A_{4}}{A_{2}+i A_{4}-k_{2}} \frac{\exp \left(-\left(A_{2}+i A_{4}+\frac{1}{\tau_{M}}\right) t\right)-\exp \left(-\frac{t}{\tau_{E 0}}\right)}{A_{2}+i A_{4}+\frac{1}{\tau_{M}}-\frac{1}{\tau_{E 0}}}\right) \\
& +k_{2}\left(\left[P y_{k 2}(D)\right]_{o}+\left[P y_{\text {diff }}(D)\right]_{o} e^{-A_{3}} \sum_{i=0}^{\infty} \frac{A_{3}^{i}}{i !} \frac{A_{2}+i A_{4}}{A_{2}+i A_{4}-k_{2}}\right) \times \frac{\exp \left(-\frac{t}{\tau_{D}}\right)-\exp \left(-\left(k_{2}+\frac{1}{\tau_{M}}\right) t\right)}{k_{2}+\frac{1}{\tau_{M}}-\frac{1}{\tau_{D}}} \\
& \left.+\left[P y_{\text {diff }}(D)\right]_{o} e^{-A_{3}} \sum_{i=0}^{\infty} \frac{A_{3}^{i}}{i !} \frac{A_{2}+i A_{4}}{A_{2}+i A_{4}-k_{2}} \frac{\exp \left(-\left(A_{2}+i A_{4}+\frac{1}{\tau_{M}}\right) t\right)-\exp \left(-\frac{t}{\tau_{D}}\right)}{A_{2}+i A_{4}+\frac{1}{\tau_{M}}-\frac{1}{\tau_{D}}}\right) \\
& +[E 0]_{o} \times \exp \left(-\frac{t}{\tau_{E 0}}\right)+[D]_{o} \times \exp \left(-\frac{t}{\tau_{D}}\right)
\end{aligned}
$$

In Equations $\mathrm{S} 3$ and $\mathrm{S} 4$, the parameters $A_{2}, A_{3}$, and $A_{4}$ are given in Equations $\mathrm{S} 5 . \mathrm{a}-\mathrm{c}$.

$$
\begin{aligned}
& A_{2}=<n>\times \frac{k_{\text {blob }} k_{e}[\text { blob }]}{k_{\text {blob }}+k_{e}[\text { blob }]} \\
& A_{3}=<n>\times\left(\frac{k_{\text {blob }}}{k_{\text {blob }}+k_{e}[\text { blob }]}\right)^{2}
\end{aligned}
$$

$$
A_{4}=k_{\text {blob }}+k_{e}[\text { blob }]
$$

Except $\tau_{\mathrm{M}}$, which was fixed in the analysis, all other parameters were fitted in a first global analysis of the pyrene monomer and excimer fluorescence decays according to the FBM with the program 
globmis90lbg. The $k_{2}$ values were then averaged and fixed in a second global analysis of the decays with the program globmis90obg. This procedure has been found to substantially narrow the error bars on the parameters $N_{\text {blob }}$ and $k_{\text {blob. }}$. The parameters were optimized according to the MarquardtLevenberg algorithm. ${ }^{3}$ 
D] Example global FBM analysis of the monomer and excimer fluorescence decays of PGly15Glu85.
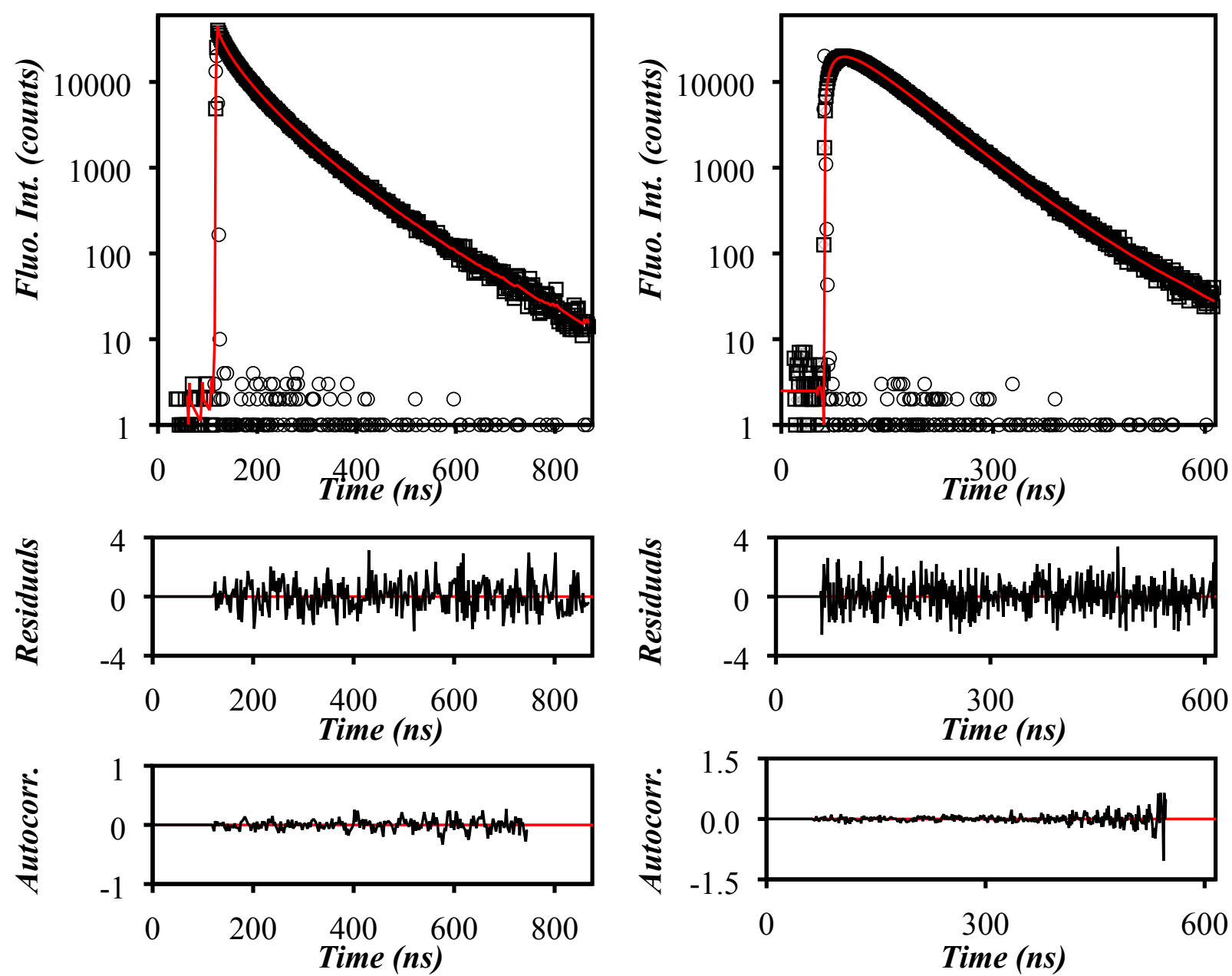

Figure S3. Monomer (left, $\lambda_{\mathrm{em}}=378 \mathrm{~nm}$ ) and excimer (right, $\left.\lambda_{\mathrm{em}}=510 \mathrm{~nm}\right)(\square)$ fluorescence decays of Py(11 mol\%)-PGly ${ }_{15} \mathrm{Glu}_{85}$ in DMSO. The (o) instrument response function and (一) fit of the global analysis of the FBM are overlaid with the fluorescence decays. $\lambda_{\mathrm{ex}}=346 \mathrm{~nm}$, $[\mathrm{Py}]=$ $2.5 \times 10^{-6}$ M. $\chi^{2}=1.04$. 


\section{E] Parameters retrieved from the FBM analysis of the pyrene-labeled polypeptides.}

Tables S1 - S3 contain the parameters retrieved from the global analysis of the monomer and excimer decays. The average value and standard deviation of the parameters determined by the error simulations described in the main text are provided in Tables $\mathrm{S} 4-\mathrm{S} 6$.

Table S1. Parameters retrieved from the FBM analysis of the pyrene monomer decays in acidified DMSO.

\begin{tabular}{|c|c|c|c|c|c|c|c|c|c|}
\hline Polypeptide & $\begin{array}{c}x \\
(\mathrm{~mol} \%)\end{array}$ & $\begin{array}{c}k_{2} \\
\left(\mathrm{~ns}^{-1}\right)\end{array}$ & $f_{\mathrm{Mk} 2}$ & $\begin{array}{c}k_{\mathrm{e}}[b l o b] \\
\left(\mu \mathrm{s}^{-1}\right)\end{array}$ & $f_{\text {Mdiff }}$ & $\begin{array}{c}k_{\text {blob }} \\
\left(\mu \mathrm{s}^{-1}\right)\end{array}$ & $<n>$ & $f_{\text {Mfree }}$ & $\chi^{2}$ \\
\hline \multirow{5}{*}{$\begin{array}{c}\text { Py-PGlu } \\
\tau_{\mathrm{M}}=170 \mathrm{~ns}\end{array}$} & 6.0 & 0.15 & 0.14 & 8.6 & 0.39 & 14.7 & 1.31 & 0.47 & 1.12 \\
\hline & 8.0 & 0.15 & 0.19 & 4.2 & 0.50 & 13.2 & 1.48 & 0.31 & 1.10 \\
\hline & 10.4 & 0.15 & 0.20 & 5.7 & 0.46 & 11.9 & 1.60 & 0.34 & 1.12 \\
\hline & 11.5 & 0.15 & 0.19 & 4.3 & 0.55 & 13.1 & 1.30 & 0.26 & 1.09 \\
\hline & 12.3 & 0.15 & 0.24 & 3.0 & 0.57 & 12.2 & 1.46 & 0.19 & 1.13 \\
\hline \multirow{5}{*}{$\begin{array}{c}\text { Py- } \\
\text { PGly }_{15} \mathrm{Glu}_{85} \\
\tau_{\mathrm{M}}=170 \mathrm{~ns}\end{array}$} & 4.0 & 0.09 & 0.15 & 3.7 & 0.55 & 6.7 & 1.26 & 0.30 & 0.98 \\
\hline & 7.6 & 0.09 & 0.24 & 3.8 & 0.69 & 6.0 & 1.68 & 0.08 & 1.09 \\
\hline & 9.0 & 0.09 & 0.30 & 2.5 & 0.67 & 6.2 & 1.90 & 0.03 & 1.06 \\
\hline & 11.0 & 0.09 & 0.35 & 3.7 & 0.63 & 6.7 & 2.32 & 0.02 & 1.04 \\
\hline & 12.6 & 0.09 & 0.44 & 3.4 & 0.55 & 6.3 & 2.77 & 0.01 & 1.06 \\
\hline \multirow{4}{*}{$\begin{array}{c}\text { Py- } \\
\text { PGly }_{40} \mathrm{Glu}_{60} \\
\tau_{\mathrm{M}}=170 \mathrm{~ns}\end{array}$} & 2.6 & 0.09 & 0.09 & 7.6 & 0.43 & 8.7 & 1.18 & 0.48 & 1.02 \\
\hline & 4.4 & 0.09 & 0.12 & 4.9 & 0.68 & 8.0 & 1.18 & 0.20 & 1.08 \\
\hline & 5.2 & 0.09 & 0.17 & 4.2 & 0.67 & 7.6 & 1.36 & 0.16 & 1.08 \\
\hline & 7.4 & 0.09 & 0.23 & 3.6 & 0.71 & 7.2 & 1.75 & 0.06 & 1.00 \\
\hline \multirow{4}{*}{$\begin{array}{c}\text { Py- } \\
\text { PGly }_{52} \mathrm{Glu}_{48} \\
\tau_{\mathrm{M}}=170 \mathrm{~ns}\end{array}$} & 2.8 & 0.14 & 0.07 & 13.8 & 0.39 & 12.4 & 1.28 & 0.54 & 1.26 \\
\hline & 3.5 & 0.14 & 0.09 & 9.3 & 0.48 & 10.1 & 1.39 & 0.43 & 1.08 \\
\hline & 4.3 & 0.14 & 0.14 & 7.0 & 0.62 & 12.7 & 1.39 & 0.24 & 1.15 \\
\hline & 5.3 & 0.14 & 0.16 & 7.3 & 0.6 & 11.5 & 1.52 & 0.24 & 1.27 \\
\hline
\end{tabular}


Table S2. Parameters retrieved using the FBM analysis of the pyrene excimer decays in acidified DMSO.

\begin{tabular}{|c|c|c|c|c|c|c|c|c|c|}
\hline Polypeptide & $\begin{array}{c}x \\
(\mathrm{~mol} \%)\end{array}$ & $f_{\mathrm{Ek} 2}$ & $\begin{array}{c}\tau_{\mathrm{E} 0} \\
(\mathrm{~ns})\end{array}$ & $f_{\text {EdiffE } 0}$ & $f_{\mathrm{EE} 0}$ & $\begin{array}{c}\tau_{D} \\
\text { (ns) }\end{array}$ & $f_{\text {EdiffD }}$ & $f_{\mathrm{ED}}$ & $\chi^{2}$ \\
\hline \multirow{5}{*}{$\begin{array}{c}\text { Py-PGlu } \\
\tau_{\mathrm{M}}=170 \mathrm{~ns}\end{array}$} & 6.0 & 0.22 & 49 & 0.64 & 0.10 & 134 & 0.02 & 0.02 & 1.12 \\
\hline & 8.0 & 0.26 & 35 & 0.24 & 0.08 & 58 & 0.41 & 0.01 & 1.10 \\
\hline & 10.4 & 0.28 & 47 & 0.59 & 0.10 & 104 & 0.04 & 0.00 & 1.12 \\
\hline & 11.5 & 0.23 & 46 & 0.57 & 0.11 & 82 & 0.08 & 0.01 & 1.09 \\
\hline & 12.3 & 0.26 & 40 & 0.41 & 0.08 & 68 & 0.21 & 0.03 & 1.13 \\
\hline \multirow{5}{*}{$\begin{array}{c}\text { Py- } \\
\text { PGly }_{15} \mathrm{Glu}_{85} \\
\tau_{\mathrm{M}}=170 \mathrm{~ns}\end{array}$} & 4.0 & 0.21 & 50 & 0.75 & 0.02 & 149 & 0.01 & 0.00 & 0.98 \\
\hline & 7.6 & 0.24 & 46 & 0.68 & 0.05 & 89 & 0.04 & 0.00 & 1.09 \\
\hline & 9.0 & 0.29 & 44 & 0.59 & 0.00 & 70 & 0.06 & 0.05 & 1.06 \\
\hline & 11.0 & 0.33 & 43 & 0.52 & 0.02 & 62 & 0.07 & 0.05 & 1.04 \\
\hline & 12.6 & 0.40 & 44 & 0.45 & 0.06 & 49 & 0.05 & 0.04 & 1.06 \\
\hline \multirow{4}{*}{$\begin{array}{c}\text { Py- } \\
\text { PGly }_{40} \mathrm{Glu}_{60} \\
\tau_{\mathrm{M}}=170 \mathrm{~ns}\end{array}$} & 2.6 & 0.16 & 52 & 0.76 & 0.05 & 148 & 0.02 & 0.01 & 1.02 \\
\hline & 4.4 & 0.14 & 55 & 0.40 & 0.00 & 34 & 0.41 & 0.05 & 1.08 \\
\hline & 5.2 & 0.19 & 48 & 0.71 & 0.05 & 89 & 0.05 & 0.00 & 1.08 \\
\hline & 7.4 & 0.23 & 42 & 0.55 & 0.00 & 61 & 0.17 & 0.06 & 1.00 \\
\hline \multirow{4}{*}{$\begin{array}{c}\text { Py- } \\
\text { PGly }_{52} \mathrm{Glu}_{48} \\
\tau_{\mathrm{M}}=170 \mathrm{~ns}\end{array}$} & 2.8 & 0.13 & 49 & 0.79 & 0.04 & 141 & 0.00 & 0.07 & 1.26 \\
\hline & 3.5 & 0.15 & 48 & 0.81 & 0.03 & 145 & 0.00 & 0.04 & 1.08 \\
\hline & 4.3 & 0.17 & 52 & 0.75 & 0.00 & 156 & 0.00 & 0.01 & 1.15 \\
\hline & 5.3 & 0.2 & 51 & 0.75 & 0.05 & 153 & 0.00 & 0.01 & 1.27 \\
\hline
\end{tabular}


Table S3. Fraction of pyrene species calculated using parameters retrieved from the FBM analysis of the pyrene monomer and excimer decays in acidified DMSO.

\begin{tabular}{|c|c|c|c|c|c|c|c|c|c|c|}
\hline Polypeptide & $\begin{array}{c}x \\
(\mathrm{~mol} \%)\end{array}$ & $f_{\mathrm{k} 2}$ & $f_{\text {diffEO }}$ & $f_{\text {diffD }}$ & $f_{\text {diff }}$ & $f_{\mathrm{E} 0}$ & $f_{\mathrm{D}}$ & $f_{\text {agg }}$ & $f_{\text {free }}$ & $\chi^{2}$ \\
\hline \multirow{5}{*}{$\begin{array}{c}\text { Py-PGlu } \\
\tau_{\mathrm{M}}=170 \mathrm{~ns}\end{array}$} & 6.0 & 0.13 & 0.36 & 0.01 & 0.37 & 0.06 & 0.01 & 0.07 & 0.44 & 1.12 \\
\hline & 8.0 & 0.18 & 0.17 & 0.29 & 0.46 & 0.06 & 0.00 & 0.06 & 0.29 & 1.10 \\
\hline & 10.4 & 0.19 & 0.40 & 0.03 & 0.43 & 0.07 & 0.00 & 0.07 & 0.31 & 1.12 \\
\hline & 11.5 & 0.18 & 0.44 & 0.06 & 0.50 & 0.08 & 0.01 & 0.09 & 0.23 & 1.09 \\
\hline & 12.3 & 0.21 & 0.34 & 0.18 & 0.52 & 0.07 & 0.03 & 0.10 & 0.17 & 1.13 \\
\hline \multirow{5}{*}{$\begin{array}{c}\text { Py-PGly }{ }_{15} \text { Glu }_{85} \\
\tau_{\mathrm{M}}=170 \mathrm{~ns}\end{array}$} & 4.0 & 0.15 & 0.53 & 0.01 & 0.54 & 0.00 & 0.02 & 0.02 & 0.30 & 0.98 \\
\hline & 7.6 & 0.23 & 0.62 & 0.03 & 0.66 & 0.00 & 0.04 & 0.04 & 0.07 & 1.09 \\
\hline & 9.0 & 0.28 & 0.57 & 0.06 & 0.63 & 0.05 & 0.00 & 0.05 & 0.03 & 1.06 \\
\hline & 11.0 & 0.33 & 0.51 & 0.07 & 0.59 & 0.05 & 0.02 & 0.07 & 0.02 & 1.04 \\
\hline & 12.6 & 0.40 & 0.45 & 0.05 & 0.50 & 0.04 & 0.06 & 0.10 & 0.01 & 1.06 \\
\hline \multirow{4}{*}{$\begin{array}{c}\text { Py- PGly }{ }_{40} \mathrm{Glu}_{60} \\
\tau_{\mathrm{M}}=170 \mathrm{~ns}\end{array}$} & 2.6 & 0.08 & 0.40 & 0.01 & 0.42 & 0.01 & 0.02 & 0.03 & 0.47 & 1.02 \\
\hline & 4.4 & 0.12 & 0.32 & 0.33 & 0.65 & 0.04 & 0.00 & 0.04 & 0.19 & 1.08 \\
\hline & 5.2 & 0.16 & 0.60 & 0.05 & 0.65 & 0.00 & 0.04 & 0.04 & 0.15 & 1.08 \\
\hline & 7.4 & 0.22 & 0.52 & 0.16 & 0.68 & 0.05 & 0.00 & 0.05 & 0.05 & 1.00 \\
\hline \multirow{4}{*}{$\begin{array}{c}\text { Py- PGly }{ }_{52} \mathrm{Glu}_{48} \\
\tau_{\mathrm{M}}=170 \mathrm{~ns}\end{array}$} & 2.8 & 0.06 & 0.38 & 0.00 & 0.38 & 0.00 & 0.03 & 0.03 & 0.52 & 1.26 \\
\hline & 3.5 & 0.09 & 0.46 & 0.00 & 0.46 & 0.00 & 0.02 & 0.02 & 0.42 & 1.08 \\
\hline & 4.3 & 0.13 & 0.58 & 0.00 & 0.58 & 0.06 & 0.01 & 0.07 & 0.22 & 1.15 \\
\hline & 5.3 & 0.15 & 0.58 & 0.00 & 0.58 & 0.03 & 0.01 & 0.04 & 0.23 & 1.27 \\
\hline
\end{tabular}


Table S4. Parameters retrieved from the error analysis of the pyrene monomer decays. The italicized numbers equal one standard deviation.

\begin{tabular}{|c|c|c|c|c|c|c|c|c|c|}
\hline Polypeptide & $x(\mathrm{~mol} \%)$ & $\begin{array}{c}k_{2} \\
\left(\mathrm{~ns}^{-1}\right)\end{array}$ & $f_{\mathrm{Mk} 2}$ & $\begin{array}{c}k_{\mathrm{e}}[\text { blob }] \\
\left(\mu \mathrm{s}^{-1}\right)\end{array}$ & $f_{\text {Mdiff }}$ & $\begin{array}{l}k_{\text {blob }} \\
\left(\mu \mathrm{s}^{-1}\right)\end{array}$ & $<n>$ & $f_{\text {Mfree }}$ & $\chi^{2}$ \\
\hline \multirow{10}{*}{$\begin{array}{c}\text { Py-PGlu } \\
\tau_{\mathrm{M}}=170 \mathrm{~ns}\end{array}$} & \multirow[t]{2}{*}{6.0} & \multirow[t]{2}{*}{0.15} & 0.136 & 8.1 & 0.394 & 14.8 & 1.32 & 0.471 & 1.01 \\
\hline & & & 0.003 & 0.7 & 0.002 & 0.5 & 0.03 & 0.003 & 0.03 \\
\hline & \multirow[t]{2}{*}{8.0} & \multirow[t]{2}{*}{0.15} & 0.189 & 4.6 & 0.500 & 14.0 & 1.42 & 0.311 & 1.10 \\
\hline & & & 0.002 & 0.1 & 0.002 & 0.3 & 0.01 & 0.001 & 0.04 \\
\hline & \multirow[t]{2}{*}{10.4} & \multirow[t]{2}{*}{0.15} & 0.205 & 5.7 & 0.455 & 11.6 & 1.63 & 0.340 & 1.00 \\
\hline & & & 0.002 & 0.6 & 0.001 & 0.6 & 0.06 & 0.002 & 0.03 \\
\hline & \multirow[t]{2}{*}{11.5} & \multirow[t]{2}{*}{0.15} & 0.200 & 4.2 & 0.541 & 12.5 & 1.33 & 0.259 & 1.06 \\
\hline & & & 0.002 & 0.1 & 0.002 & 0.2 & 0.01 & 0.001 & 0.03 \\
\hline & \multirow[t]{2}{*}{12.3} & \multirow[t]{2}{*}{0.15} & 0.251 & 2.8 & 0.562 & 11.6 & 1.48 & 0.186 & 1.08 \\
\hline & & & 0.001 & 0.1 & 0.002 & 0.1 & 0.02 & 0.003 & 0.04 \\
\hline \multirow{10}{*}{$\begin{array}{c}\text { Py-PGly }{ }_{15} \text { Glu }_{85} \\
\tau_{\mathrm{M}}=170 \mathrm{~ns}\end{array}$} & \multirow[t]{2}{*}{4.0} & \multirow[t]{2}{*}{0.09} & 0.150 & 3.2 & 0.558 & 6.5 & 1.27 & 0.292 & 0.99 \\
\hline & & & 0.003 & 0.2 & 0.008 & 0.4 & 0.08 & 0.007 & 0.03 \\
\hline & \multirow[t]{2}{*}{7.6} & \multirow[t]{2}{*}{0.09} & 0.233 & 3.7 & 0.690 & 6.26 & 1.63 & 0.077 & 1.01 \\
\hline & & & 0.001 & 0.1 & 0.002 & 0.09 & 0.02 & 0.003 & 0.03 \\
\hline & \multirow[t]{2}{*}{9.0} & \multirow[t]{2}{*}{0.09} & 0.302 & 2.6 & 0.666 & 6.2 & 1.92 & 0.032 & 1.01 \\
\hline & & & 0.001 & 0.2 & 0.003 & 0.1 & 0.03 & 0.003 & 0.03 \\
\hline & \multirow[t]{2}{*}{11.0} & \multirow[t]{2}{*}{0.09} & 0.355 & 3.5 & 0.625 & 6.4 & 2.38 & 0.020 & 1.03 \\
\hline & & & 0.002 & 0.2 & 0.002 & 0.1 & 0.03 & 0.001 & 0.03 \\
\hline & \multirow[t]{2}{*}{12.6} & \multirow[t]{2}{*}{0.09} & 0.445 & 3.3 & 0.545 & 6.1 & 2.84 & 0.010 & 1.16 \\
\hline & & & 0.002 & 0.4 & 0.001 & 0.2 & 0.07 & 0.001 & 0.03 \\
\hline \multirow{8}{*}{$\begin{array}{c}\text { Py- } \text { PGly }_{40} \text { Glu }_{60} \\
\tau_{\mathrm{M}}=170 \mathrm{~ns}\end{array}$} & \multirow[t]{2}{*}{2.6} & \multirow[t]{2}{*}{0.09} & 0.089 & 8.0 & 0.427 & 8.7 & 1.20 & 0.484 & 1.03 \\
\hline & & & 0.001 & 0.2 & 0.001 & 0.1 & 0.02 & 0.001 & 0.03 \\
\hline & \multirow[t]{2}{*}{4.4} & 0.09 & 0.151 & 5.69 & 0.646 & 7.3 & 1.18 & 0.203 & 1.10 \\
\hline & & & 0.001 & 0.03 & 0.001 & 0.1 & 0.02 & 0.002 & 0.06 \\
\hline & 5.2 & 0.09 & 0.173 & 3.8 & 0.670 & 7.32 & 1.39 & 0.157 & 1.09 \\
\hline & & & 0.001 & 0.2 & 0.003 & 0.09 & 0.02 & 0.003 & 0.04 \\
\hline & 7.4 & 0.09 & 0.238 & 3.3 & 0.703 & 6.8 & 1.81 & 0.059 & 1.05 \\
\hline & & & 0.002 & 0.2 & 0.003 & 0.1 & 0.02 & 0.002 & 0.05 \\
\hline & 2.8 & 0.14 & 0.066 & 13 & 0.393 & 12.8 & 1.28 & 0.541 & 1.03 \\
\hline & & & 0.002 & 1 & 0.002 & 0.9 & 0.07 & 0.002 & 0.03 \\
\hline & 3.5 & 0.14 & 0.089 & 9.5 & 0.480 & 10.4 & 1.37 & 0.431 & 1.03 \\
\hline Py- PGly $_{52} \mathrm{Glu}_{48}$ & & & 0.001 & 0.5 & 0.001 & 0.5 & 0.04 & 0.002 & 0.03 \\
\hline & 4.3 & 0.14 & 0.139 & 7.2 & 0.619 & 13.0 & 1.38 & 0.241 & 1.06 \\
\hline & & & 0.001 & 0.1 & 0.001 & 0.1 & 0.01 & 0.001 & 0.01 \\
\hline & 5.3 & 0.14 & 0.159 & 7.6 & 0.599 & 11.7 & 1.51 & 0.241 & 1.04 \\
\hline & & & 0.001 & 0.2 & 0.001 & 0.2 & 0.01 & 0.001 & 0.03 \\
\hline
\end{tabular}


Table S5. Parameters retrieved from the error analysis of the pyrene excimer decays. The italicized numbers equal one standard deviation.

\begin{tabular}{|c|c|c|c|c|c|c|c|}
\hline Polypeptide & $x(\mathrm{~mol} \%)$ & $f_{\mathrm{Ek} 2}$ & $f_{\text {Ediff }}$ & $f_{\text {Eagg }}$ & $\begin{array}{c}\tau_{\mathrm{E} 0} \\
(\mathrm{~ns})\end{array}$ & $\begin{array}{c}\tau_{D} \\
(\mathrm{~ns})\end{array}$ & $\chi^{2}$ \\
\hline \multirow{10}{*}{$\begin{array}{c}\text { Py-PGlu } \\
\tau_{\mathrm{M}}=170 \mathrm{~ns}\end{array}$} & \multirow[t]{2}{*}{6.0} & 0.223 & 0.649 & 0.13 & 50.0 & 134 & 1.01 \\
\hline & & 0.004 & 0.008 & 0.01 & 0.8 & 0 & 0.03 \\
\hline & \multirow[t]{2}{*}{8.0} & 0.249 & 0.660 & 0.091 & 37.8 & 57.0 & 1.10 \\
\hline & & 0.002 & 0.008 & 0.004 & 0.8 & 0.2 & 0.04 \\
\hline & \multirow[t]{2}{*}{10.4} & 0.28 & 0.64 & 0.11 & 46.5 & 105 & 1.00 \\
\hline & & 0.09 & 0.04 & 0.08 & 0.6 & 9 & 0.03 \\
\hline & \multirow[t]{2}{*}{11.5} & 0.235 & 0.64 & 0.13 & 45.3 & 84 & 1.06 \\
\hline & & 0.002 & 0.02 & 0.02 & 0.9 & 7 & 0.03 \\
\hline & \multirow[t]{2}{*}{12.3} & 0.271 & 0.607 & 0.122 & 36.2 & 60.4 & 1.08 \\
\hline & & 0.002 & 0.006 & 0.004 & 0.3 & 0.3 & 0.04 \\
\hline \multirow{10}{*}{$\begin{array}{c}\text { Py-PGly15Glu85 } \\
\tau_{\mathrm{M}}=170 \mathrm{~ns}\end{array}$} & \multirow[t]{2}{*}{4.0} & 0.208 & 0.77 & 0.021 & 50 & 110 & 0.99 \\
\hline & & 0.004 & 0.01 & 0.005 & 1 & 30 & 0.03 \\
\hline & \multirow[t]{2}{*}{7.6} & 0.24 & 0.86 & 0.05 & 43.2 & 89.4 & 1.01 \\
\hline & & 0.07 & 0.02 & 0.05 & 0.4 & 0.9 & 0.03 \\
\hline & \multirow[t]{2}{*}{9.0} & 0.30 & 0.76 & 0.05 & 43.1 & 67 & 1.01 \\
\hline & & 0.07 & 0.04 & 0.06 & 0.4 & 1 & 0.03 \\
\hline & \multirow[t]{2}{*}{11.0} & 0.34 & 0.67 & 0.07 & 42.1 & 58 & 1.03 \\
\hline & & 0.07 & 0.09 & 0.09 & 0.5 & 4 & 0.03 \\
\hline & \multirow[t]{2}{*}{12.6} & 0.402 & 0.493 & 0.106 & 43.4 & 49 & 1.16 \\
\hline & & 0.001 & 0.007 & 0.002 & 0.2 & 0 & 0.03 \\
\hline \multirow{8}{*}{$\begin{array}{c}\text { Py- PGly40Glu60 } \\
\tau_{\mathrm{M}}=170 \mathrm{~ns}\end{array}$} & \multirow[t]{2}{*}{2.6} & 0.162 & 0.774 & 0.064 & 51.5 & 155 & 1.03 \\
\hline & & 0.001 & 0.002 & 0.002 & 0.4 & 4 & 0.03 \\
\hline & \multirow[t]{2}{*}{4.4} & 0.177 & 0.76 & 0.068 & 49.6 & 34 & 1.10 \\
\hline & & 0.001 & 0.01 & 0.001 & 0.1 & 0 & 0.06 \\
\hline & \multirow[t]{2}{*}{5.2} & 0.194 & 0.753 & 0.053 & 47.3 & 89 & 1.09 \\
\hline & & 0.001 & 0.005 & 0.006 & 0.3 & 0 & 0.04 \\
\hline & \multirow[t]{2}{*}{7.4} & 0.237 & 0.70 & 0.06 & 31.4 & 47.1 & 1.05 \\
\hline & & 0.002 & 0.02 & 0.02 & 1.5 & 0.3 & 0.05 \\
\hline \multirow{8}{*}{$\begin{array}{c}\text { Py- PGly52Glu48 } \\
\tau_{\mathrm{M}}=170 \mathrm{~ns}\end{array}$} & \multirow[t]{2}{*}{2.8} & 0.128 & 0.76 & 0.11 & 51.2 & 147 & 1.03 \\
\hline & & 0.003 & 0.02 & 0.02 & 1.1 & 5 & 0.03 \\
\hline & \multirow[t]{2}{*}{3.5} & 0.146 & 0.783 & 0.071 & 48.7 & 150 & 1.03 \\
\hline & & 0.002 & 0.006 & 0.009 & 0.8 & 6 & 0.03 \\
\hline & \multirow[t]{2}{*}{4.3} & 0.18 & 0.80 & 0.01 & 52.3 & 160 & 1.06 \\
\hline & & 0.04 & 0.04 & 0.04 & 0.3 & 10 & 0.01 \\
\hline & \multirow[t]{2}{*}{5.3} & 0.20 & 0.74 & 0.06 & 51.2 & 160 & 1.04 \\
\hline & & 0.04 & 0.03 & 0.05 & 0.2 & 10 & 0.03 \\
\hline
\end{tabular}


Table S6. Global parameters retrieved from the error analysis of the pyrene monomer and excimer decays. The italicized numbers equal one standard deviation.

\begin{tabular}{|c|c|c|c|c|c|c|c|}
\hline Polypeptide & $\begin{array}{c}x \\
(\mathrm{~mol} \%)\end{array}$ & $N_{\text {blob }}$ & $f_{\mathrm{k} 2}$ & $f_{\text {diff }}$ & $f_{\text {agg }}$ & $f_{\text {free }}$ & $\chi^{2}$ \\
\hline \multirow{10}{*}{$\begin{array}{c}\text { Py-PGlu } \\
\tau_{\mathrm{M}}=170 \mathrm{~ns}\end{array}$} & \multirow{2}{*}{6.0} & 11.7 & 0.126 & 0.366 & 0.072 & 0.437 & 1.01 \\
\hline & & 0.3 & 0.003 & 0.002 & 0.002 & 0.003 & 0.03 \\
\hline & \multirow[t]{2}{*}{8.0} & 12.3 & 0.176 & 0.468 & 0.065 & 0.291 & 1.10 \\
\hline & & 0.1 & 0.001 & 0.002 & 0.003 & 0.002 & 0.04 \\
\hline & \multirow[t]{2}{*}{10.4} & 10.4 & 0.190 & 0.422 & 0.072 & 0.315 & 1.00 \\
\hline & & 0.4 & 0.001 & 0.002 & 0.003 & 0.002 & 0.03 \\
\hline & \multirow[t]{2}{*}{11.5} & 8.6 & 0.180 & 0.488 & 0.098 & 0.234 & 1.06 \\
\hline & & 0.1 & 0.002 & 0.003 & 0.003 & 0.002 & 0.03 \\
\hline & \multirow[t]{2}{*}{12.3} & 9.8 & 0.2258 & 0.505 & 0.102 & 0.167 & 1.08 \\
\hline & & 0.1 & 0.0008 & 0.001 & 0.003 & 0.003 & 0.04 \\
\hline \multirow{10}{*}{$\begin{array}{c}\text { Py-PGly15Glu } 85 \\
\tau_{\mathrm{M}}=170 \mathrm{~ns}\end{array}$} & \multirow[t]{2}{*}{4.0} & 23 & 0.148 & 0.550 & 0.0146 & 0.287 & 0.99 \\
\hline & & 1 & 0.003 & 0.008 & 0.0005 & 0.007 & 0.03 \\
\hline & \multirow[t]{2}{*}{7.6} & 19.8 & 0.222 & 0.656 & 0.049 & 0.073 & 1.01 \\
\hline & & 0.2 & 0.001 & 0.002 & 0.002 & 0.003 & 0.03 \\
\hline & \multirow[t]{2}{*}{9.0} & 20.6 & 0.2865 & 0.631 & 0.052 & 0.030 & 1.01 \\
\hline & & 0.3 & 0.0007 & 0.002 & 0.003 & 0.003 & 0.03 \\
\hline & \multirow[t]{2}{*}{11.0} & 21.2 & 0.329 & 0.579 & 0.07 & 0.019 & 1.03 \\
\hline & & 0.2 & 0.001 & 0.003 & 0.01 & 0.001 & 0.03 \\
\hline & \multirow[t]{2}{*}{12.6} & 22.3 & 0.398 & 0.488 & 0.105 & 0.009 & 1.16 \\
\hline & & 0.5 & 0.001 & 0.002 & 0.002 & 0.001 & 0.03 \\
\hline \multirow{8}{*}{$\begin{array}{c}\text { Py- PGly40Glu60 } \\
\tau_{\mathrm{M}}=170 \mathrm{~ns}\end{array}$} & \multirow[t]{2}{*}{2.6} & 23.8 & 0.0863 & 0.412 & 0.0340 & 0.468 & 1.03 \\
\hline & & 0.3 & 0.0008 & 0.001 & 0.0008 & 0.001 & 0.03 \\
\hline & \multirow[t]{2}{*}{4.4} & 21.4 & 0.1430 & 0.6103 & 0.055 & 0.192 & 1.10 \\
\hline & & 0.4 & 0.0009 & 0.0007 & 0.001 & 0.002 & 0.06 \\
\hline & \multirow[t]{2}{*}{5.2} & 22.6 & 0.165 & 0.640 & 0.045 & 0.150 & 1.09 \\
\hline & & 0.3 & 0.001 & 0.003 & 0.001 & 0.003 & 0.04 \\
\hline & \multirow[t]{2}{*}{7.4} & 23.0 & 0.224 & 0.660 & 0.061 & 0.055 & 1.05 \\
\hline & & 0.2 & 0.002 & 0.003 & 0.002 & 0.002 & 0.05 \\
\hline \multirow{8}{*}{$\begin{array}{c}\text { Py- PGly52Glu48 } \\
\tau_{\mathrm{M}}=170 \mathrm{~ns}\end{array}$} & \multirow[t]{2}{*}{2.8} & 21.0 & 0.062 & 0.372 & 0.053 & 0.513 & 1.03 \\
\hline & & 1.2 & 0.002 & 0.001 & 0.002 & 0.003 & 0.03 \\
\hline & \multirow[t]{2}{*}{3.5} & 22.3 & 0.085 & 0.4596 & 0.042 & 0.413 & 1.03 \\
\hline & & 0.7 & 0.001 & 0.0009 & 0.002 & 0.002 & 0.03 \\
\hline & \multirow[t]{2}{*}{4.3} & 24.3 & 0.1378 & 0.612 & 0.011 & 0.2387 & 1.06 \\
\hline & & 0.2 & 0.0008 & 0.001 & 0.002 & 0.0005 & 0.01 \\
\hline & \multirow[t]{2}{*}{5.3} & 21.6 & 0.1514 & 0.570 & 0.049 & 0.2296 & 1.04 \\
\hline & & 0.2 & 0.0007 & 0.001 & 0.002 & 0.0006 & 0.03 \\
\hline
\end{tabular}




\section{F] Circular Dichroism of the pyrene-labeled polypeptides.}

The molar ellipticity $([\theta])$ of the pyrene-labeled polypeptides studied in the main text are plotted as a function of wavelength in Figures S4B - E. Note the absence of peaks near the ${ }^{1} B_{b}$ and ${ }^{1} L_{a}$ bands of pyrene (Figure S4A). In contrast, the CD spectra of pyrene-labeled poly( $L$-glutamic acid) (Py-P ${ }_{L}$ Glu) in Figure S4F, which adopts a $3{ }_{10}$-helical structure in DMSO, ${ }^{4,5}$ exhibits strong peaks near the absorption bands of pyrene. 

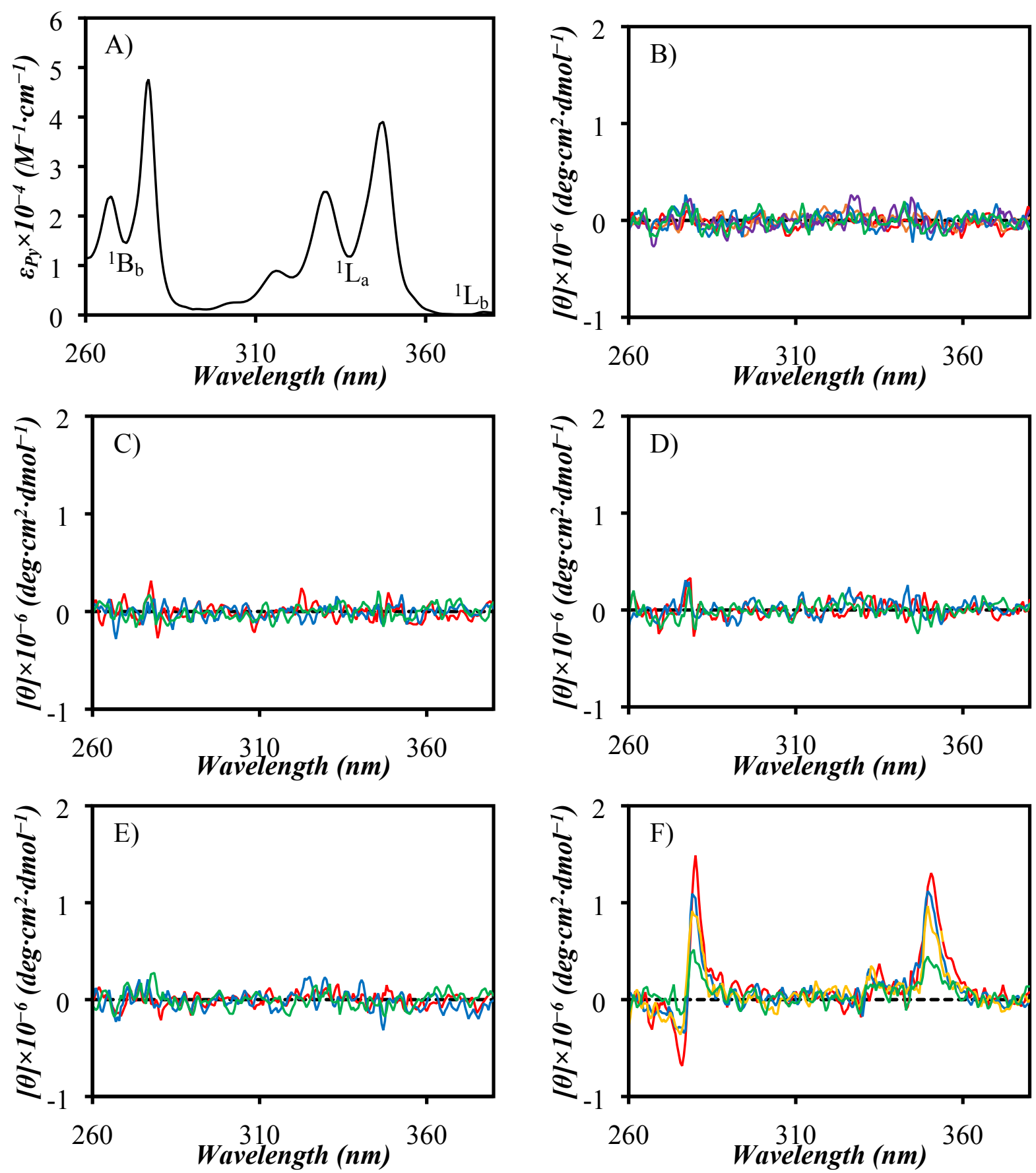

Figure S4. A) An example absorption spectrum of the pyrene-labeled polypeptides in DMSO. The molar ellipticity of B) Py-PGlu $(x=(-) 6.0,(-)$ 8.0, $(-)$ 0.4, $(-)$ 11.5, and (-) $12.3 \mathrm{~mol} \%)$, C) Py-PGly $_{15} \mathrm{Glu}_{85}\left(x=(-)\right.$ 4.0, (-) 9.0, and (-) 12.6 mol\%), D) Py-PGly ${ }_{40} \mathrm{Glu}_{60}(x=(-)$ 2.6, (一) 5.2, and (-) $7.4 \mathrm{~mol} \%$ ), E) Py-PGly ${ }_{52} \mathrm{Glu}_{48}(x=(-)$ 2.8, (-) 3.5, and (-) $5.3 \mathrm{~mol} \%$ ), and F) Py-P ${ }_{L}$ Glu $(x=(-) 4.9,(-)$ 9.0, (-) 14.3, and (-) $18.4 \mathrm{~mol} \%)$ in DMSO. 
For the sake of completeness, the CD spectra of pyrene-labeled poly $(D, L$-alanine-co- $D, L$ glutamic acid) (Py-PAlaGlu) and poly( $D, L-N_{6}$-carbobenzyloxylysine-co- $D, L$-glutamic acid) (PyPLys(Z)Glu), which were assumed to adopt coiled conformations in a previous study² in DMSO are provided in Figures S5A and B. The absence of peaks in Figures S5A and B demonstrate that the previously studied Py-PAlaGlu's and Py-PLys(Z)Glu's were indeed unstructured in DMSO.
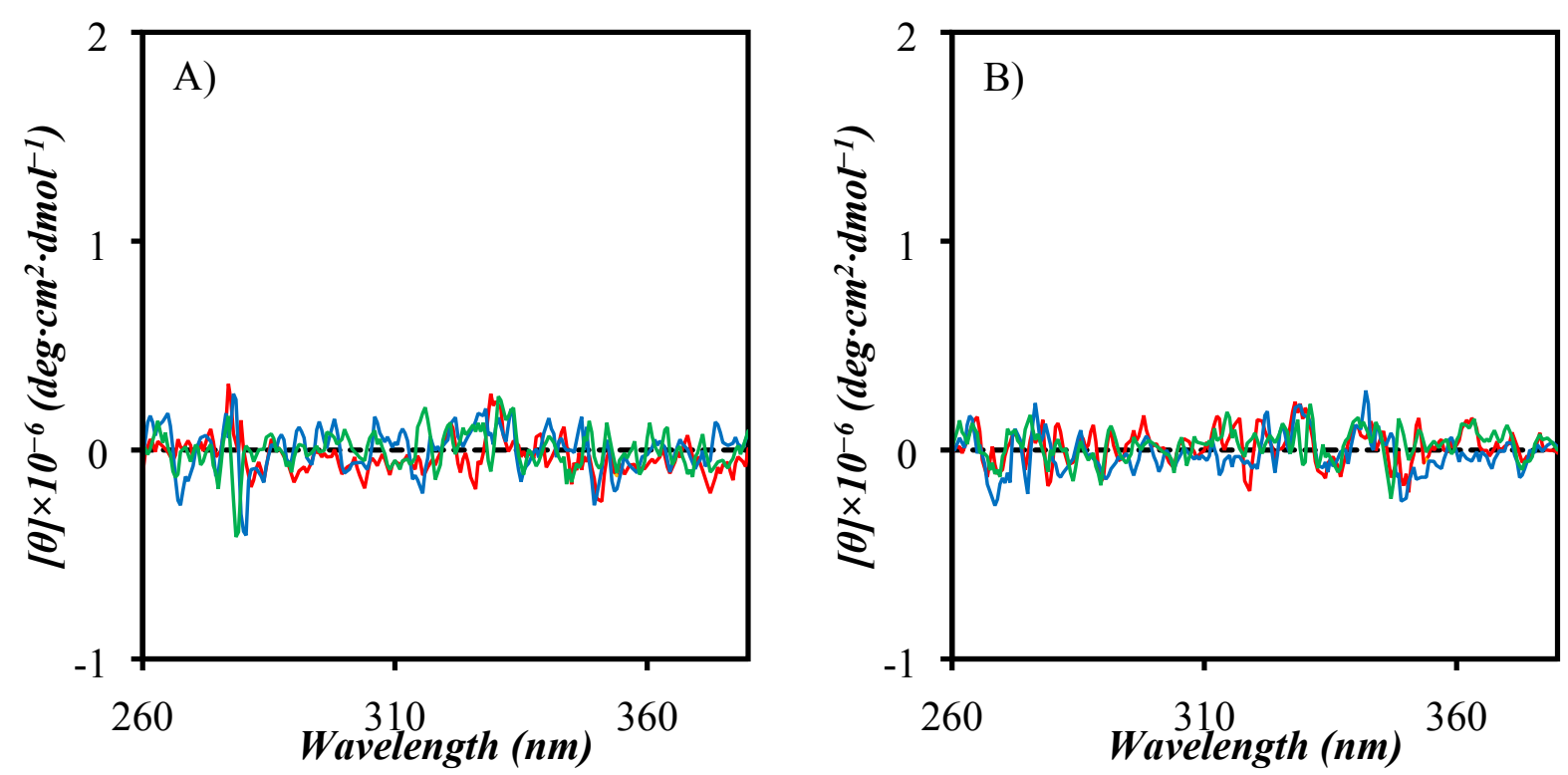

Figure S5. CD spectra of A) Py-PAlaGlu $\left(f_{\text {Ala }}=58 \mathrm{~mol} \%, x=(-) 6.6,(-)\right.$ 13.5, and (-) 15.2 mol\%) and B) Py-PLys $(Z) G l u\left(f_{\mathrm{Lys}(\mathrm{Z})}=57 \mathrm{~mol} \%, x=(-) 5.5,(-) 10.0\right.$, and $\left.(-) 13.2 \mathrm{~mol} \%\right)$ in DMSO. 
G] Fluorescence spectra of the Py-PGlu, Py-PGly ${ }_{40} \mathrm{Glu}_{60}$, and Py-PGly ${ }_{52} \mathrm{Glu}_{48}$ samples.
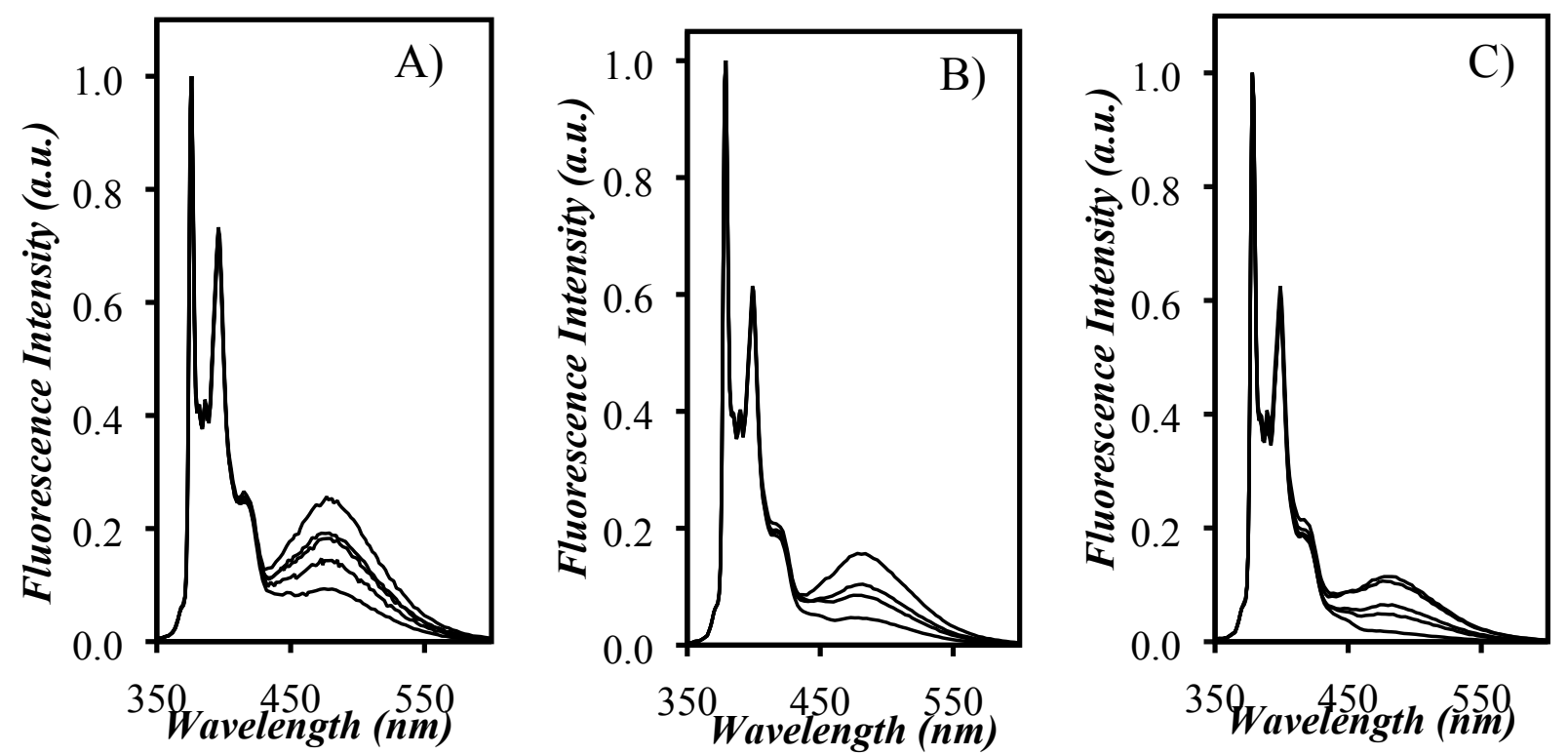

Figure S6. Steady-state fluorescence spectra of A) Py-PGlu, B) Py-PGly ${ }_{40} \mathrm{Glu}_{60}$, and C) PyPGly ${ }_{52} \mathrm{Glu}_{48}$ in DMSO. $\lambda_{\mathrm{ex}}=346.5 \mathrm{~nm}$. 
H] $k_{\text {blob }}$ values of the pyrene-labeled polypeptides.

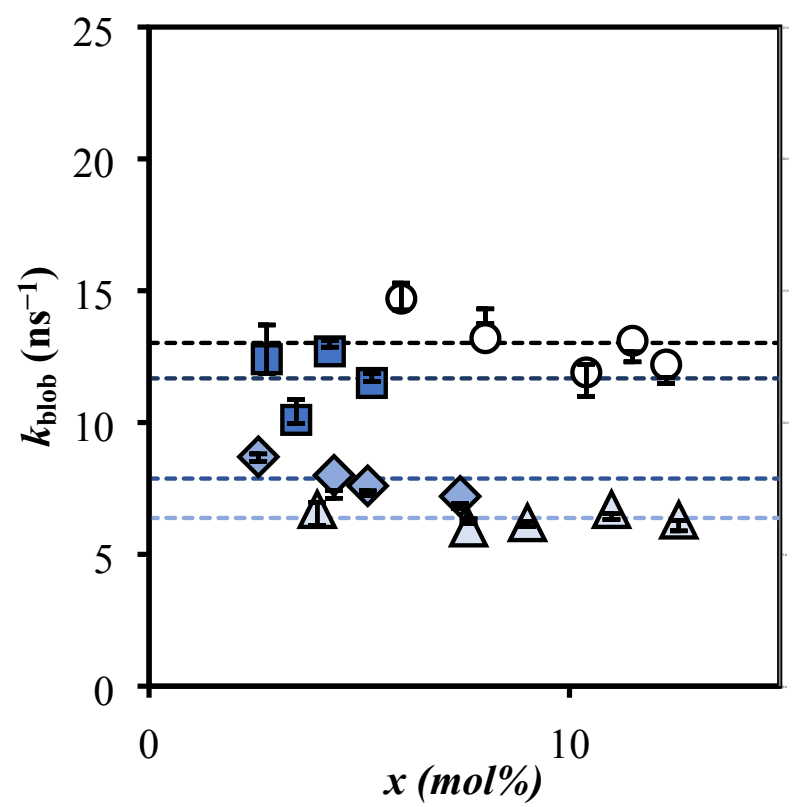

Figure S7. Plot of $k_{\text {blob }}$ values as a function of pyrene content for $(O)$ Py-PGlu, $(\diamond)$ Py$\operatorname{PGly}_{15} \mathrm{Glu}_{85},(\Delta) \mathrm{Py}_{-\mathrm{PGly}}{ }_{40} \mathrm{Glu}_{60}$, and $(\square)$ Py-PGly ${ }_{52} \mathrm{Glu}_{48}$. The dashed lines represent the average $<k_{\text {blob }}>$ values. The error bars (one $\sigma$ ) were retreived from the error analysis described in the main text. 


\section{I] References}

1. Casier, R.; Duhamel, J. Pyrene Excimer Fluorescence as a Direct and Easy Experimental Means to Characterize the Length Scale and Internal Dynamics of Polypeptide Foldons. Macromolecules 2018, 51, 3450-3457.

2. Casier, R.; Duhamel, J. The Effect of Amino Acid Size on the Internal Dynamics and Conformational Freedom of Polypeptides. Macromolecules 2020, 53, 9811-9822.

3. Press, W. H.; Flanery, B. P.; Tenkolsky, S. A.; Vetterling, W. T. Numerical Recipes in Fortran: The Art of Scientific Computing; Cambridge University Press: Cambridge and New York, 1992; pp $523-528$.

4. Berbeć, S.; Dec, R.; Molodenskiy, D.; Wielgus-Kutrowska, B; Johannessen, C.; HernikMagoń, A.; Tobias, F.; Bzowska, A; Ścibisz, G.; Keiderling, T. A.; Svergun, D.; Dzwolak, W. ß2-Type Amyloidlike Fibrils of Poly-l-glutamic Acid Convert into Long, Highly Ordered Helices upon Dissolution in Dimethyl Sulfoxide. J. Phys. Chem. B 2018, 122, 11895-11905.

5. Casier, R; Duhamel, J. Effect of like Charges on the Conformation and Internal Dynamics of Polypeptides Probed by Pyrene Excimer Fluorescence. Macromolecules 2020, 53, 51475157. 